\title{
Rechtsgeschichte
}

http://www.rg-rechtsgeschichte.de/rg4

$\operatorname{Rg} 2004$

$217-220$

Zitiervorschlag: Rechtsgeschichte Rg 4 (2004)

http://dx.doi.org/10.12946/rg04/217-220

\section{Frank Rexroth}

\section{Eintausendzweihundertdreiundsiebzig Sheriffs hatten keine Frau}


gewesen, sondern im Licht der seit dem I2. Jahrhundert entwickelten kanonistischen Schuldlehre als surprisingly old-fashioned.

Ein vergleichbar geringes Interesse für die juristischen Verfahrensgrundlagen zeigt Jeffrey Denton in seiner Darstellung des nie zu Ende geführten Prozesses gegen Papst Bonifaz VIII. (I3O3-I3I2). Die Vermutung, dem französischen König Philipp dem Schönen sei es um die Beherrschung einer »public opinion « gegangen, weil in den Prozessakten immer wieder die »publica fama " genannt ist, ist ein Paradebeispiel dafür, auf welche Irrwege gerät, wer meint, Prozessakten auch ohne den Umweg zeitraubender Vorarbeiten sachgerecht beurteilen zu können: Der »fama « wiesen weltliche und kirchliche Autoren der kontinentalen gelehrten Prozessliteratur einen festen Platz innerhalb der hierarchischen Beweislehre zu und behandelten sie gar im Rahmen monographischer Traktate. So erklärt das mittelalterliche gelehrte Beweisrecht, nicht aber ein Bedürfnis nach »controlling of public opinion «, die gänzlich konventionelle Omnipräsenz der »fama publica « in den von Philipp dem Schönen und seinen Räten gegen Bonifaz VIII. entworfenen Beweisartikeln - wie der Autor überdies dem von ihm zitierten Standardwerk Tilmann Schmidts zum Bonifaz-Prozess ohne weiteres hätte entnehmen können.

Insgesamt hinterlässt der Band einen zwiespältigen Eindruck. Trotz einer Zahl anregender Beiträge verhindert die willkürliche Auswahl der Themen, das Überwiegen von Spezialfragen und der nahezu durchgängige Verzicht auf grundlegende Informationen zur Gerichtsverfassung und auf Erläuterung der komplexen Terminologie des englischen Prozessrechts dem Werk den Charakter "grundlegend « zuzusprechen. Aber welcher Tagungsband hätte dieses Prädikat schon verdient?

Thomas Wetzstein

\section{Eintausendzweihundertdreiundsiebzig Sheriffs hatten keine Frau*}

Man muss kein Vielschreiber sein, um eine historische Meistererzählung aus den Angeln zu heben. Der Oxforder Historiker K. B. McFarlane schaffte dies mit seiner enormen Begeisterungsfähigkeit als akademischer Lehrer. Der whiggistischen Ansicht, dass man die englische Geschichte von der Magna Carta bis zum Beginn der Lancasterherrschaft (und dann abermals von den Tudors an) als die kontinuierliche Rationalisierung des Verhältnisses von Krone und Parlament beschreiben kann, setzte der Oxforder Historiker eher ein Forschungsprogramm für

\begin{abstract}
künftige Generationen denn eine Alternativerzählung entgegen. Sein Grundgedanke bestand darin, dass sich die politische Dimension der englischen Geschichte bei weitestgehender Konzentration auf Krone, Hof und Parlament nicht erschöpfend beschreiben lässt, sondern dass ein Studium der lokalen Elitekulturen in den Grafschaften konstitutiv für ein Verständnis der Akteure und ihres politischen Horizonts sei. Dies eröffnete in der Tat neue Horizonte für viele, denn gefragt waren damit empirische Studien von einer gewissen Tiefenschärfe, Prosopogra-
\end{abstract}

\footnotetext{
* Richard Gorski, The Fourteenth-Century Sheriff. English Local Administration in the Late Middle Ages, Woodbridge, Rochester, N. Y.: The Boydell Press $2003, \mathrm{X}, 2 \mathrm{I} 3 \mathrm{~S}$., ISBN 0-8 5 I I 5-933-8
} 
phien und Erhebungen komplizierter Besitzstruktur, das Studium der adligen Grundherrschaften und ihrer Heiratsverbindungen. Auf diesem Weg wurde jedoch das alte Paradigma >Repräsentation und Konstitutionalismus unversehens gegen neue Leitvorstellungen ausgetauscht: `Klientelismus< als Strategie der großen Familien, die lokale Gentry an sich zu binden und so ihre Machtposition zu arrondieren; >Patronage $<$ und >Dienst< als reziprokes Verhältnis, das den Hoch- und den Niederadel aneinander band; ’Bastardfeudalismus` als die Möglichkeit, die Ritterschaft mit Hilfe vertraglicher Abmachungen auf Zeit und gegen Geldleistungen zu Privatarmeen zusammenzuführen. Die Ämter der Administration in den Grafschaften erschienen unter diesem Vorzeichen wie ein Feld, auf dem der König und die lokalen Magnaten um die Macht im Lande konkurrierten.

Doch während der vergangenen zehn Jahre wurde die Publikation immer neuer "gentry studies « auch kritisch kommentiert. Dabei ging es nicht so sehr um die empirische Seite dieser Arbeiten. Vielmehr wuchs das Unbehagen mit dem zugrunde liegenden Interpretament einer geschlossenen "county community«. Das Bedürfnis nach einer neuen Perspektive entstand, die die Lokalstudien gemeinsam mit Arbeiten zu Monarchie und Hof sowie denen zu den politischen Aspirationen abseits des Adels zu einer Geschichte der politischen Kultur Englands zu vereinen helfen würde.

Richard Gorski, der Autor einer empiriegesättigten neuen Monographie, hat die Botschaft nur zu Teilen vernommen. Was seine Studie auszeichnet, ist die Entscheidung, den Rahmen der Regionalstudie aufzugeben und die Geschichte der englischen Sheriffs im I4. Jahrhundert für das gesamte Königreich zu schreiben. Was ihr wie so vielen vorangegangenen Arbeiten fehlt, ist ein Politikmodell, das Monarchiegeschichte und Adelsgeschichte, die Mentalität des Landbesitzes und die der rationalisierten Verwaltung, insbesondere aber Konsens und Konflikt zu integrieren statt gegeneinander auszuspielen vermag. Gorski hatte zunächst von der Zuversicht gezehrt, dass man McFarlanes Anliegen im Zeitalter der Datenbank im ganz großen Rahmen einlösen, dass man der gesamtenglischen Gentry mittels der Aggregation möglichst umfassender Datensätze ihre bisher verborgenen Geheimnisse entlocken könne. Nach einigen Jahren Arbeit zügelte er seinen Ehrgeiz und entschied sich, auf der Grundlage des bisher erhobenen Materials lediglich eine Studie zum Sheriffsamt als dem prominentesten Posten vorzulegen, der in den einzelnen Grafschaften zu vergeben war. Auf diese Weise will er nun zeigen, wie die Personen, die im Auftrag der Krone auf begrenzte Zeit Aufgaben wahrnehmen, doppelt vernetzt waren: horizontal in ihren Statusgruppen, vertikal in klientelistischen Bindungen mit Hochadel, König oder beiden. Entschieden wendet er sich gegen die geläufige Annahme, dass die Sheriffs lediglich durch ihren Landbesitz für ihr Amt empfohlen wurden und dass die Übernahme administrativer Aufgaben als Beiwerk und Verbrämung einer im Innersten von aristokratischen Werten regierten Elite anzusehen sei. Seine Analyse von Amtsinhabern, so Gorski, belege stattdessen, dass die Sheriffs des I4. Jahrhunderts eine Funktionselite gebildet hätten. Freilich sei es hilfreich gewesen, über erheblichen Grundbesitz zu verfügen - dieser steigerte ganz offensichtlich ihre Akzeptanz in einer Bevölkerung, die von Fremden und Aufsteigern in erster Linie persönliche Bereicherung, Amtsmissbrauch und Ausbeutung erwartete. Doch auch Erfahrung, Kompetenz und Motivation habe den guten Sheriff ausgezeichnet. Wer woll- 
te da widersprechen? Dass Expertise und Durchsetzungsvermögen nicht schaden konnten, galt sicher für alle Ämter, die die Krone temporär zu vergeben hatte - sei es die Nominierung zum Steuereintreiber, Coroner oder Escheator oder auch die Delegation in die Friedenskommissionen und das Parlament.

Zwischen I 300 und I 400 wurden insgesamt I 273 Personen zu Sheriffs ernannt und somit zu Experten für die Verhaftung Verdächtiger, das Eintreiben von Leistungen für den König, die Aushebung und Inspektion von Truppen und den Beistand für andere Amtsträger gemacht. Unter der Botmäßigkeit der Krone befanden sich die Inhaber dieses ursprünglich angelsächsischen Amtes schon seit den Kindertagen des Lehnsstaates: Fiskalisch unterstanden sie dem königlichen Exchequer, judiziell den königlichen Richtern und personell der Krone selbst. Schon im I2. und I3. Jahrhundert hatten die Könige ihre Sheriffs nicht mehr aus der Gruppe der Barone rekrutiert, sondern sich verstärkt auf Ritterschaft und die darunter angesiedelte »squirearchy « verlassen. Für Maitland und andere hatte es daher nahe gelegen, die Geschichte dieses Amts als Verlustgeschichte zu schreiben - eine Sicht, die nicht unbestritten blieb und die Gorski nun gänzlich zu widerlegen sucht. Zwar scheint ihm das I4. Jahrhundert die konsequente Fortsetzung älterer Tendenzen zu bringen, doch belegt er zugleich, dass stets dann Umdefinitionen des Amtes anstanden, wenn die Autorität der Krone grundsätzlich in Frage gestellt wurde; so I3 I I mit der Tätigkeit der Ordainers, I340/4I während Edwards III. Revirement seines Apparats, abermals in dessen letzten Jahren und in den

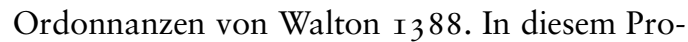
zess wurde die jährliche Neuvergabe des Amtes ebenso durchgesetzt, wie man mit einem Iterationsverbot experimentierte.
Wenn die Sheriffs nicht durchweg, aber doch dominierend der lokalen Gentry entstammten und wenn in der Forschung bisher Konsens darüber bestand, dass diese über Grundbesitz, Kriegsdienst, Lebensstil und die Verwurzelung in der lokalen Elite (»territoriality «) definiert sei - wie sichert man eine These wie diejenige $a b$, dass persönliche Befähigung und Bereitschaft zum schnöden administrativen Engagement entscheidend für die Wahl der Amtsträger sei? Auf empirischem Weg ist dies nur schwerlich möglich, denn was besagt es schon, dass die Amtsinhaber in vielen Fällen ein zweites und drittes Mal ins Amt berufen wurden oder dass viele von ihnen noch im hohen Alter ihren Dienst versahen? Steht Alter notwendigerweise für Erfahrung? Alle diese quantifizierbaren Angaben sind mit Akribie zusammengetragen worden, aber wie mag man sie bewerten? Wenn Gorski anmahnt, man solle über der Präponderanz der Ritterschaft nicht die wenigen unter-ritterlichen Sheriffs übersehen - was ändert dies dann an dem bestehenden Gesamtbild? Noch am ehesten in seinem Teilkapitel über das Bild vom Sheriff in der volkssprachlichen fiktionalen Literatur vergisst er seinen ausgeprägten Kliometrismus und vermag zu zeigen, dass die soziale Schätzung der Sheriffs durch den reichsweiten Diskurs über Korruption und Amtsmissbrauch bestimmt wurde. Wenig überzeugend erscheint daher Gorskis Tendenz, die Bindungen zwischen den Akteuren in Zentrale und Region im Vokabular von »Partnerschaft « einzufangen - Krone und »local communities «, Ritter und Esquires - sie alle waren Partner, und der Sheriff stand im Zentrum dieser wunderbaren Beziehungen. Seine Vergemeinschaftung zu studieren, ihm bei der Kommunikation mit den Aristokraten und den Angehörigen der königlichen Zentralgewalt zuzuhören, 
ist in Gorskis Untersuchung nicht angelegt. Die I 273 Sheriffs des I4. Jahrhunderts haben in ihrer Datenbank ein einsames Dasein geführt; sie wurden zwar ernannt und verfügten über Grundbesitz und, sofern erschließbar, über ein Alter nach Jahren, kannten aber keine Verwandtschaftsbande und blieben daher derjenigen sozialen Dimensionen beraubt, die ihre politischen Selbstzuordnungen gesteuert haben werden. Sie scheinen nicht geheiratet, sich verschwägert, sich potenter Gevatter versichert zu haben; die einzige Frau im ganzen Buch ist eine Hochadlige, die den Sheriff zu Hilfe gerufen hat. Die Rede von der »local community « wird auf diese Weise zu einer Leerformel. Sollte es daran liegen, dass Prosopographie hier nicht als Methode zur Einlösung eines definierten Erkenntnisinteresses betrachtet wird, sondern als ein oberhalb der Probleme und Debatten beheimateter Königsweg, so als könne man mit ihrer Hilfe die Wirklichkeit in ihrer Totalität erfassen? Gorski begreift die Verwaltungsgeschichte Englands als eine Summe jeweils personenbezogener Daten zu Ernennung, Vermögen, Ämteriteration und Alter. Die Sozialgeschichte der Sheriffs jedoch, verstanden als Geschichte ihrer Vergemeinschaftung, wird die Forschung auch weiterhin beschäftigen.

Frank Rexroth

\section{Auf der Suche nach einer Ordnung des Rechts*}

Italien ist offenbar ein fruchtbarer Boden für Lehrbücher der Rechtsgeschichte. Klassisch war auch in diesem Land, wie in Deutschland und anderswo, für Lehrbücher, Vorlesungen und Lehrstühle die Zuordnung einerseits zum Römischen Recht, andererseits zur "nationalen « Rechtsgeschichte, hier also Storia del diritto italiano. So heißt auch noch die Professur von Italo Birocchi an der Sapienza in Rom. Die Bezeichnungen der Vorlesungen und der Lehrbücher haben sich jedoch in Italien in den letzten Jahrzehnten radikal geändert. Es handelte sich dabei um eine schnelle und koordinierte Reaktion der italienischen Hochschullehrer auf die Umgestaltung der juristischen Universitätsausbildung in ihrem Lande. Auf diese Studienreformen (deren neueste, auf die europäische Angleichung orientierte, gerade im Gange ist) haben die italienischen Rechtshistoriker mit einer Ausdif- ferenzierung der rechtshistorischen Vorlesungen, und zumeist mit einer dem entsprechenden Vermehrung der rechtshistorischen Professuren geantwortet - also etwa das Gegenteil dessen, was an deutschen Fakultäten der Fall war und ist. Das zeugt von der Kraft der kulturellen Überzeugung, dass die Rechtswissenschaft ein humanistisches Fach sei und nicht in erster Linie die Vermittlung normativ-technischer Kenntnisse und Fertigkeiten zum Ziele habe. Das italienische Studienprogramm ist abgedeckt durch ein aufwändiges universitäres Examenssystem, welches an das Ende jeder Vorlesung eine meist mündliche Prüfung jedes Studenten setzt, der sich durch das Bestehen einen Baustein für das Abschlussexamen erwirbt. Dies entspricht bekanntlich dem napoleonisch-französischen im Gegensatz zum deutschen Humboldtschen Universitäts- und Studienmodell. Es setzt zumeist

\footnotetext{
* Italo Birocchi, Alla Ricerca dell'Ordine. Fonti e Cultura Giuridica nell'Età Moderna (Il Diritto nella Storia 9), Torino: G. Giappichelli Editore 2002, XVI, 654 S., ISBN 88-348-22I7-X
}

ণัญ

Dilcher, Auf der Suche nach einer Ordnung des Rechts 